\title{
Procrastination on Social Networking Sites: Combating by Design
}

\author{
Abdulaziz Alblwi, Angelos Stefanidis, Keith Phalp, Raian Ali \\ Bournemouth University, UK \\ \{aalblwi, astefanidis, kphalp, rali\}@ bournemouth.ac.uk
}

\begin{abstract}
Procrastination refers to a voluntary postponement that prevents people from performing their tasks and can hurt productivity and wellbeing. Procrastination might occur due to a lack of motivation to perform tasks or due to the low self-control that people might have over their time and task management. Social Networking Sites (hereafter SNS) are designed to enable their users to engage in online interaction for different purposes such as increasing popularity or exploring information. SNS embed influence and persuasion techniques to attract users which can make them a medium for procrastination where some users fail to maintain a desirable level of self-control over their usage. However, we argue that advances in persuasive technology and gamification techniques can be utilised to augment SMS and help users to regain self-control over their procrastination. Implementing these techniques correctly means that users can still enjoy accessing SNS while maintaining a desirable level of control over their procrastination. Building these antiprocrastination tools, however, is a challenging design activity due to their potential of triggering negative side-effects such as reactance and workarounds, and affecting the overall user experience. In this paper, we conduct user studies, consisting of an exploratory stage using focus groups, diary study and interviews and followed by a design stage based mainly on codesign sessions. Our studies' participants self-declared having a problematic degree of procrastination on SNS, to explore procrastination countermeasure techniques that can augment the future designs of SNS and how best to apply them.
\end{abstract} design

Keywords- Procrastination, Social Networking design, Co-

\section{INTRODUCTION}

Procrastination refers to the voluntary delay of urgent tasks which might lead to negative consequences such as low productivity or anxiety [1]. Delays caused by procrastination can negatively impact students' academic performance (among other groups) and their learning achievement [1]. However, it has been demonstrated that managed procrastination can help people to relieve stress and improve their mood which can positively increase work efficiency [2]. With managed procrastination, people maintain good control over their procrastination and control excessive negative effects, for example by being able to resume their work at an appropriate time. However, some people fail to control their procrastination due to several factors such as their low selfcontrol and external social pressure. Procrastination is often motivated by the desire to avoid a task and through interaction with others on SNS. Hence, the process can induce stress which might be detrimental to their productivity [3].

SNS such as Twitter and YouTube enable their users to create online profiles and communicate and maintain contact with others. Despite these perceived benefits, some usage styles might turn out to be harmful to those who do not maintain good control over their usage. SNS are designed to encourage further engagement which can eventually lead users to procrastinate if this engagement conflicts with the other tasks. SNS also provide a medium for peer pressure, whereby users feel the urge to engage in developing a positive selfimage or because of their fear of missing out (FoMO) [4].

Persuasive technology and gamification are powerful tools that can help users to gain more control over their procrastination. Persuasive technology is used to help change people's behaviour through influence rather than hard coercion and pressure [5]. Gamification is the use of game elements in non-game contexts [6]. Using these techniques can be potentially useful, by motivating users to maintain better control over their usage [7]. For example, users can utilise SNS usage feedback by observing the time spent and the frequency of interaction with SNS daily. This helps to raise user's awareness about their usage and encourage them to take action to control their levels of usage better. Arguably, users might procrastinate because of the rewards that SNS offer to enhance the perceived social interaction opportunities, in the form of "likes" or "positive comments." In such cases, the use of gamification can also help bring the reward that users experience into the tasks being delayed so that users can feel more motivated to keep working on their tasks to gain further rewards such as points.

Elsewhere, persuasive technology and gamification tools demonstrate their power to motivate users to change their behaviour [8, 9]. Importantly, incorrectly embedding these tools in the design of SNS can be detrimental to the user experience and create problems [10,11]. For example, users might get overloaded when they get detailed usage feedback which might lead them to misunderstand the provided data. Gamification elements such as timers and progress bars can also lead to reactance and encourage a workaround, e.g. using a different account and device. Gamification tools can also negatively affect users' intrinsic motivation and make them more interested in the rewards they can get by following a certain behaviour $[11,12]$ than in the genuine or initial goal. 
This reinforces the necessity to define users' needs to minimise the negative side-effects that can occur when implementing these tools into SNS.

To achieve that, we conducted an exploratory stage based on focus groups, a diary study and interviews and then used the results as a basis for a follow-up co-design study to explore different countermeasures that can help users to gain greater control over their procrastination. The method allowed end users to participate in deciding what type of countermeasures to introduce to SNS and combat their procrastination and how best to apply them. The participants in our studies declared to procrastinate frequently on SNS. We report on the findings and provide a conceptualisation of procrastination and the socio-technical measures which can combat it. We also compare our findings with emerging tools in this area and propose enhancements.

The paper is organised as follows. In the next section, we present our research method. In Section III we discuss the procrastination types and the socio-technical countermeasures that can combat it and how best to apply them. In Section IV we present the challenges to deal with when applying these countermeasures and compare our findings with existing tools. Finally, in Section V, we conclude the paper and present our future work.

\section{RESEARCH METHOD}

We adopted a qualitative method to investigate procrastination on social media in-depth and followed the exploration phase with co-design sessions. This helped to understand procrastination relation to the current design of social media and explore and develop socio-technical countermeasures that can be used to combat it. In co-design and participatory design methods in general, end users can participate in creating the solutions based on their requirements, still based on theoretical foundations communicated and utilised by the researcher [13]. Co-design also refers to the creative process that is conducted collaboratively by researchers and end users to reach appropriate solutions. In our study, this process involves discussion and negotiation on how to embed some techniques into the future design of SNS to combat procrastination. However, there was a need to identify the role of SNS in leading or triggering users to procrastinate before identifying the countermeasures that can help combat it. Therefore, we first adopted the exploration stage to demonstrate how procrastination occurred and obtained the participants' views on that.

\section{A. Exploration stage}

In this stage, we followed a qualitative method to understand the perceived role of SNS features design in encouraging users to procrastinate. The exploration stage was divided into three phases: a focus group, a diary study, and follow-up interviews. The focus group collected the perceptions of the influence of SNS features concerning procrastination. The selection criteria involved participants who had at least one social network account and had also selfdeclared that they significantly experience procrastination on SNS. We recruited sixteen participants (nine female and seven male, aged over eighteen). Convenience sampling was used to recruit the participants [14]. The focus group was divided into two sessions, each session had eight participants. Subsequently, we designed a diary study that sought to elaborate on the results of the focus group over ten days. The diary method was chosen because it facilitates the collection of participants views at different times and in different contexts, hence enhancing the credibility of the findings of the focus group [15]. Then three participants were called for further clarification of the data that had been provided. This also helped as a member checking technique to validate our analysis with the subjects. This exploratory phase was required as the first step before designing the solutions that can help users to combat their procrastination. Understanding the role of SNS features in encouraging users' procrastination helps us to take a further step to search for solutions. Therefore, we followed this phase by conducting co-design sessions with end users to figure out how we can add an intervention into the future design of SNS to help users gain more control over their procrastination.

\section{B. Co-Design stage}

In this stage, we set out to identify the countermeasures techniques that can be used in future designs of SNS in order to help users to combat their procrastination. We recruited new fourteen participants (six female and eight male) into two design sessions with each session having seven participants. We used the same inclusion criteria that had been used in the previous stage for participant recruitment. Furthermore, we aimed to explore the modality of how these countermeasure techniques can be seamlessly implemented and integrated into SNS without affecting the user experience. We adopted a codesign method to collect the participant views and preferences on the design and increase the chance of its acceptance [16]. An example of the design suggested by the study participants is presented in Fig.1. The research benefits from using a codesign approach because it leads to better understanding of the end user needs, and can increase the opportunity to provide stronger solutions due to the different ideas that can be analysed, and evaluated before the implementation stage takes place [17].

We adopted three techniques during the co-design sessions in order to enhance the credibility of the study and to ensure that data bias was eliminated. The co-design sessions techniques include a standard personal introduction, storytelling and brainstorming.

1) Standard personal introduction: We gave a short presentation on procrastination and an overview regarding how SNS features encourage users to procrastinate as concluded through the first exploratory stage. This helped to immerse the participants in the research problem and involved a warming-up activity in getting the participants involved in the design sessions.

2) Storytelling: The participants were provided with different stories describing how procrastination occurs and when and why it can be problematic. The stories provided a strong sense of realism of the problem and encouraged the careful consideration of innovative solutions to cater to different contexts and usage styles. 


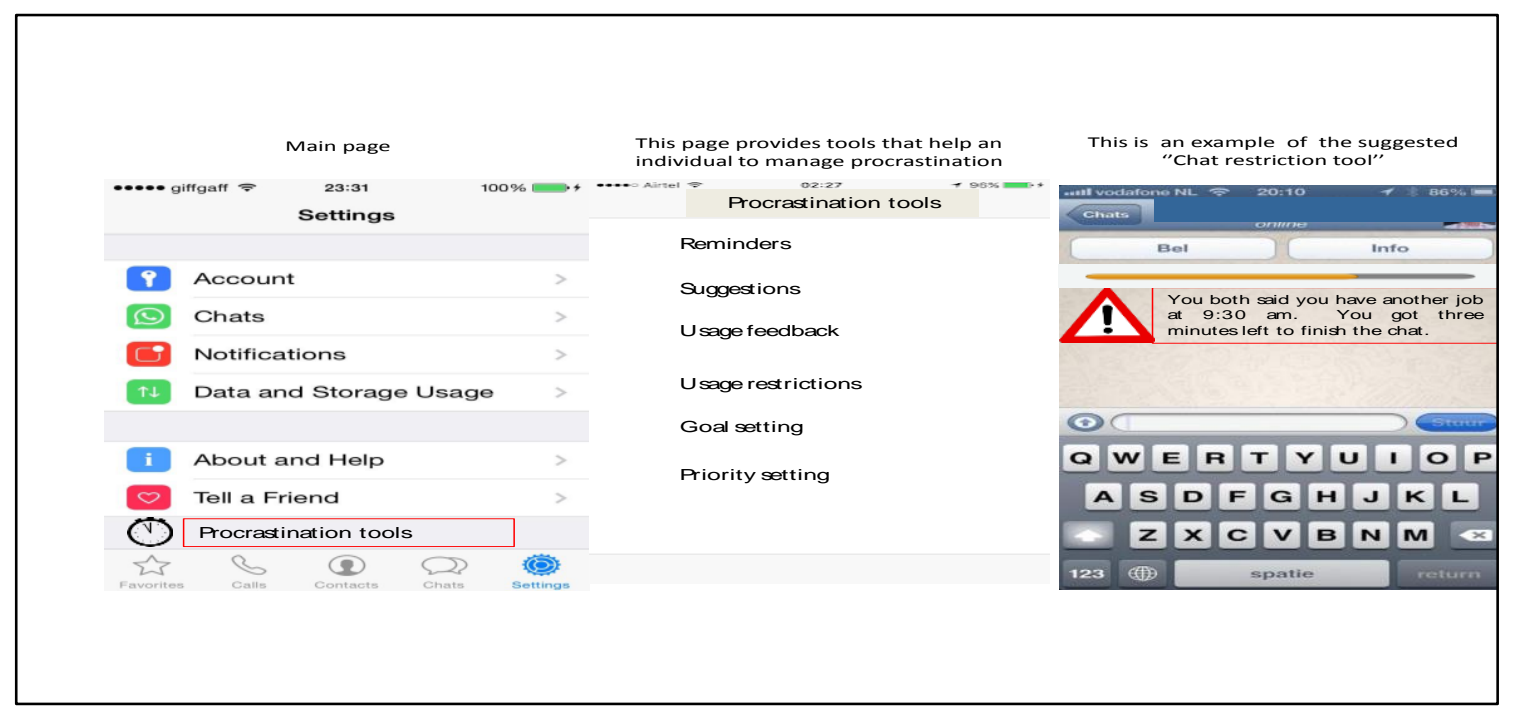

Fig.1: An example of the suggested designs during the co-design sessions

3) Brainstorming: Using scenarios provided by the research team, the participants were encouraged to think and share their ideas openly with others. These ideas were critically analysed and evaluated by the participants in order to formulate robust solutions. The participants were also asked to think about the tools they would like to see in future software designs to combat procrastination. We asked two main questions at this stage:

- What are the different techniques that can be embedded into future designs of SNS to combat procrastination?

- How can we embed the countermeasure techniques into SNSs design without affecting the user experience?

Participants provided solutions based on de-facto techniques used in current technology for different purposes including scheduling software. We abstracted them to families and categories of countermeasures. To this end, we adopted a thematic analysis method to formulate that. Thematic analysis is used to search for themes that emerge with the description of the study problems in qualitative data. We followed the six steps that were suggested in [18] to conduct the analysis. Besides, using thematic analysis helped us to categories the countermeasures techniques into different themes.

\section{DESIGN COUNTERMEASURES AND MODALITIES OF APPLICATION}

In this section, we categorise the countermeasure techniques into three distinct types: socio-technical, technical, and increasing task motivation. These types help to illustrate the different types of countermeasures that can be embedded in the future SNS design. Technical and socio-technical countermeasures seek to combat procrastination once users start accessing SNSs, while task motivation aims to prevent procrastination from occurring by motivating users to keep working on their original tasks. Moreover, in this section, we discuss how to embed the countermeasure into SNS design without adversely affecting the user experience.

\section{A. Procrastination: Socio-technical Countermeasures}

In this section, we present countermeasures that can be used to combat procrastination. This includes goal setting and calendar integration, see Fig. 2. Socio-technical countermeasures focus on the relation among the senders, recipients, and the device.

1) Goal setting: it refers to the development of an action plan that motivates individuals to follow and achieve the desired goal [19]. Goal setting is considered an effective strategy for encouraging users to change their behaviour and empowerment in terms of having more control over their SNS usage [20]. Hence, having goals and discovering what motivates users to achieve these goals, will guide users through the process of the goals achievements. Goal setting involves steps which guide users to set their goals and increase the possibility of achieving them. Goal settings process typically follows five main guidelines: setting a specific goal, defining measures, ensuring goals can be attained and relevant to the person and having sufficient time to achieve [21]. Following these steps can reduce the tendency to procrastinate and can help users of SNS to gain more control over accomplishing their goals. However, in the design sessions participants claimed that they usually access SNS without knowing how they are going to spend their time or have a clear idea of how much time they are going to spend on SNS. This leads to procrastination which causes users to neglect their original tasks. One of the participants of the study said that "the future design of SNSs should include a part to help users set their goals and keep reminding them about their goals to avoid procrastination." Other participants discussed how setting goals could be implemented within the design of SNS. Based on those suggestions, the design features were divided into two sub-categories: priority, and goal declaration.

a) Priority: When users have multiple goals to accomplish at the same time, they can decide the time they 


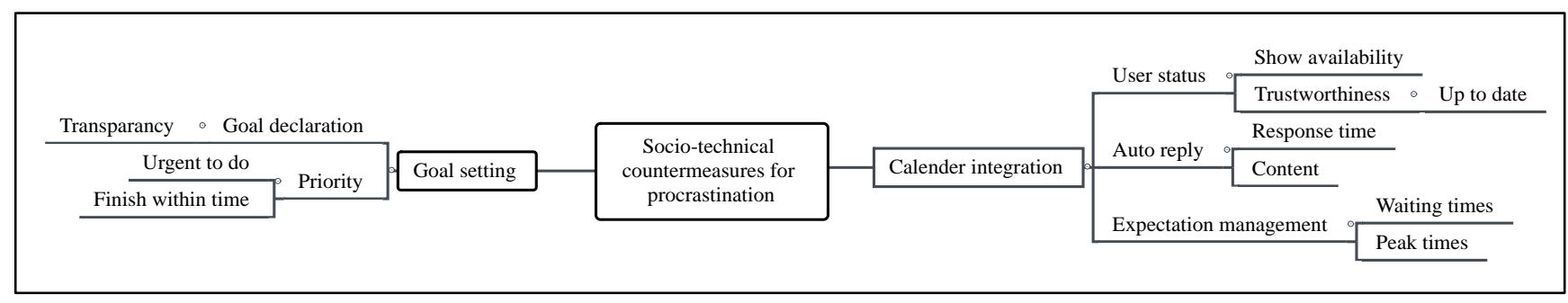

Fig. 2: Procrastionation on SNS: socio-technical countermeasures

need to accomplish each task and determine the level of urgency for each task. This can help users to determine which goals are more important and why clearly. Setting goal priorities helps users to track their progress and avoid spending time on non-urgent or unimportant tasks. Goal priority can help users to take control of their actions by determining the design process towards accomplishing their goals. It has been shown that people who have a specific goal to focus on paying more attention to it than a collection of goals, which leads to better performance compared to others who work without setting a specific goal $[19,22]$. Therefore, the urgency level and time frame allocated can help to determine the priority of the tasks.

Concerning how to implement the priority technique into the future design of SNS, the findings of the design sessions suggested that the urgency level of the goal needs to be considered when setting goals so that users can decide the level of goal priority and whether it is an urgent goal. If users have more than one goal, the priority technique can use the urgency level to decide which goal users should receive more motivation to complete. Therefore, based on the degree of urgency, users can receive reminders and suggestions about their goals to motivate them for goals achievement and at the same time avoid information overload and irrelevant or less interesting reminders. A participant said that "the app can allow users to list their priorities and based on that automated suggestions can be sent".

Finishing within time is another factor that was suggested by the participants in the design sessions to help users to determine their priorities. It is also seen helpful for users to accomplish their goals successfully and minimise the threat of procrastination. Setting a suitable timeframe for specific goals can help users to monitor their progress, while it helps to complete the task within the allotted timeframe. It has been found that people who have poor time management behaviour, such as leaving tasks at the last minute, experience workrelated stress and low academic performance [23, 24].

b) Goal declaration: Declaring goals to contacts and colleagues can help users to avoid getting pressure when they ignore other users comments and requests on SNS. For example, when a user receives a notification or a message while performing a particular task, it may cause them to procrastinate by feeling the urge to check and respond in order to maintain a good relationship with others and build a positive self-image despite having other active goals and tasks disrupted. Goals declaration as a countermeasure can help users to avoid procrastination by showing user' contacts the availability time of the user. Hence, users can avoid procrastination and still build a positive self-image when they fulfil their promises and respond once they get free time. A user can have the option that allows them to be more transparent and provide more details about their goals to inspire trust. One participant said: "I usually procrastinate by responding to my contacts' requests on SNS to maintain good relations with them. If they realise that I am busy they will not distract me",

2) Calendar integration: The calendar integration is one of the countermeasures that were proposed by the participants of the design sessions to help them to combat their procrastination on SNSs. The calendar integration technique is divided into three sub-categories: user status, auto-reply, and expectation management.

a) User status: User status represents the present situation of an individual on SNS when those users are active. The status can also be used to share certain information with others such as user availability. However, participants suggested that the visibility of user status to show the availability of the user can help to combat procrastination on SNS. User status can indicate to other users whether the user is available or busy. However, some users tend to use the same status for a long time without making a change which often harms the trustworthiness of the status feature. One participant said: "I have seen people who have not changed their status for more than three years." This has a significant effect in terms of trusting the status of those users, rendering it irrelevant. Therefore, user status can be integrated with the user's calendar to keep it updated to increase the likelihood of others trusting it and avoiding distraction which will, in turn, lead to reduced procrastination.

b) Autoreply: The autoreply technique can help to reduce the pressure that the recipients and the sender to manage their expectations of each other well in terms of response time and also priorities, e.g. "I am dealing with the high workload at the moment. I may take longer to reply to your message". This technique manages senders' expectations because they can know when they might receive responses. As a result, the autoreply can help in the optimisation of time management for both senders and recipients. Autoreply is divided into two subcategories: response time and content. Both categories have different benefits in terms of empowering the autoreply technique to help users gain more control over their procrastination.

Response time refers to how fast other users can receive the autoreply and whether the autoreply can be sent instantly upon receiving a notification or if it is controlled by the users themselves. It was debated at length in the design sessions of this study whether an instant automated reply can help both 
the sender to avoid procrastination more than the controlled reply due to the time that the user spends thinking about whether or not to send the reply, depending on the message received. One participant said: "I would not use the autoreply if I received an important notification." Meanwhile, some participants suggested that "Using autoreply to tell others about the time that they can get a response help to avoid procrastination and reduce the pressure that senders might have to interact". They can still override that if the message is important.

Autoreply content and its framing can have a significant effect on the recipients' reaction and belief in the message. The content can be personalised or general. For example, each user can receive an autoreply that includes their name so that user might feel their request has been given more attention. In the general content, all users can receive the same content on the automated reply.

c) Expectation management: Users who use SNS to maintain or seek popularity might use SNS excessively to meet others' expectations and avoid disappointing them which can result in procrastination. However, managing the sender's expectations can reduce the pressure that the receiver feels when they receive a notification through. For example, when a user receives a notification, the sender is unaware whether the receiver is available. This might put some pressure on the receiver to access their SNS and interact, which might lead to procrastination. Therefore, the sender's expectations can be managed better if the sender knows more details about the time that they can expect a reply. This can be achieved by considering certain factors that include the length of time to wait before getting a response, and the peak time at which the user is usually busy.

Concerning the modality of the calendar integration, the participants suggested that SNSs should be integrated with the user's online calendar or the to-do-list and the goals that users declared and based on that the user status will suggest whether the user is available. This technique can help to manage senders' expectations and reduce the pressure that receivers feel when they receive a notification enticing them to interact. Also, the auto-reply can be customised based on the closeness of contact and other criteria and preferences. For example, the user can customise the autoreply for those who are deemed to be important or close to them but provide more general content for others. One participant said: "I prefer to generate the autoreply to have some more information about me to some contacts so they will know I appreciate their request."

\section{B. Technical countermeasures}

This section presents the technical countermeasures designed to help users combat their procrastination. These countermeasures focus on the relation between the user and their device. The technical countermeasures were divided into three sub-categories that include reminders, self-monitoring, and suggestions (see Fig.3).

1) Reminder: Is a persuasive technique that can be used to help users tackle their procrastination on SNSs [25]. Reminder techniques can be integrated with the user goals or calendars, to send reminders that motivate users to have more control over their procrastination. When users have planned a change in their behaviour and set up measures and tools for that, including a reminder, it suggests that they have the intention to achieve that change. Behaviour change literature claims that initiating and strengthening the intentional goal is an important factor in transforming these goal intentions into actual behaviour [26]. The ease of implementing these intentions depend on how confident people are about their ability to transform these intentions into actual behaviour [27]. For example, when a user intends to stop procrastinating while working on their original tasks, they are more likely to do so when they are confident about their ability to control their usage. Therefore, the reminder technology helps remind the users about their intentions when they deviate from it, e.g., by sending them an alert or vibration. The strength of the user's intention can be increased when the time of reminders and their frequency are sensitive to the user's context. Users can be reminded about various responsibilities such as a subject reminder, usage reminder, and context sensitivity reminder.

a) Subject reminder: With the subject reminder, users can set a reminder about the tasks that need to be performed. For example, users procrastinating on SNS can receive reminders which help to raise their awareness and motivate them to stop procrastinating. Users can be reminded either about current tasks, new tasks, or alternative content. The participants claimed that users can have the option to decide how they would like to receive reminders, whether in the form of vibrations, sounds or changing the screen colour, e.g. using green, amber, red lights.

b) Usage reminder: The usage reminders can be used to remind users about the time they tend to spend on SNS. For example, when a user accesses an SNS, the reminder technique will monitor their usage and issue reminders accordingly. Users will have the ability to design those reminders and their frequency. Hence, based on usage, the number of reminders can be increased, and the way of delivering those reminders can be changed. For example, users can receive a reminder if they spend more than 30 minutes on SNS and then a new reminder after a further 15 minutes, with another after a further 5 minutes. Increasing the number of reminders would lead to alarming users and result in them focusing on the importance to stop to procrastinate. A participant said that "the frequency of the reminders and the content can strongly affect users decisions to stop procrastination". This can be debated as sending multiple reminders can also lead to ignoring the initial reminders expecting another reminder to come close to the deadline.

c) Context sensitivity: Some users can set a reminder based on their location, such as being on campus and having to attend a lecture. The reminder technique can integrate with the user's location and different reminders can be sent when the user procrastinates. The context sensitivity reminder might appear immediately when a user accesses their SNS in a certain context and let them know that SNS access conflicts with their expected tasks in that context. The users can design the content of the message they would like to receive the reminder when they access SNS in particular locations. The ability to choose what users would like to receive can help to minimise any side-effects associated with the messages and 


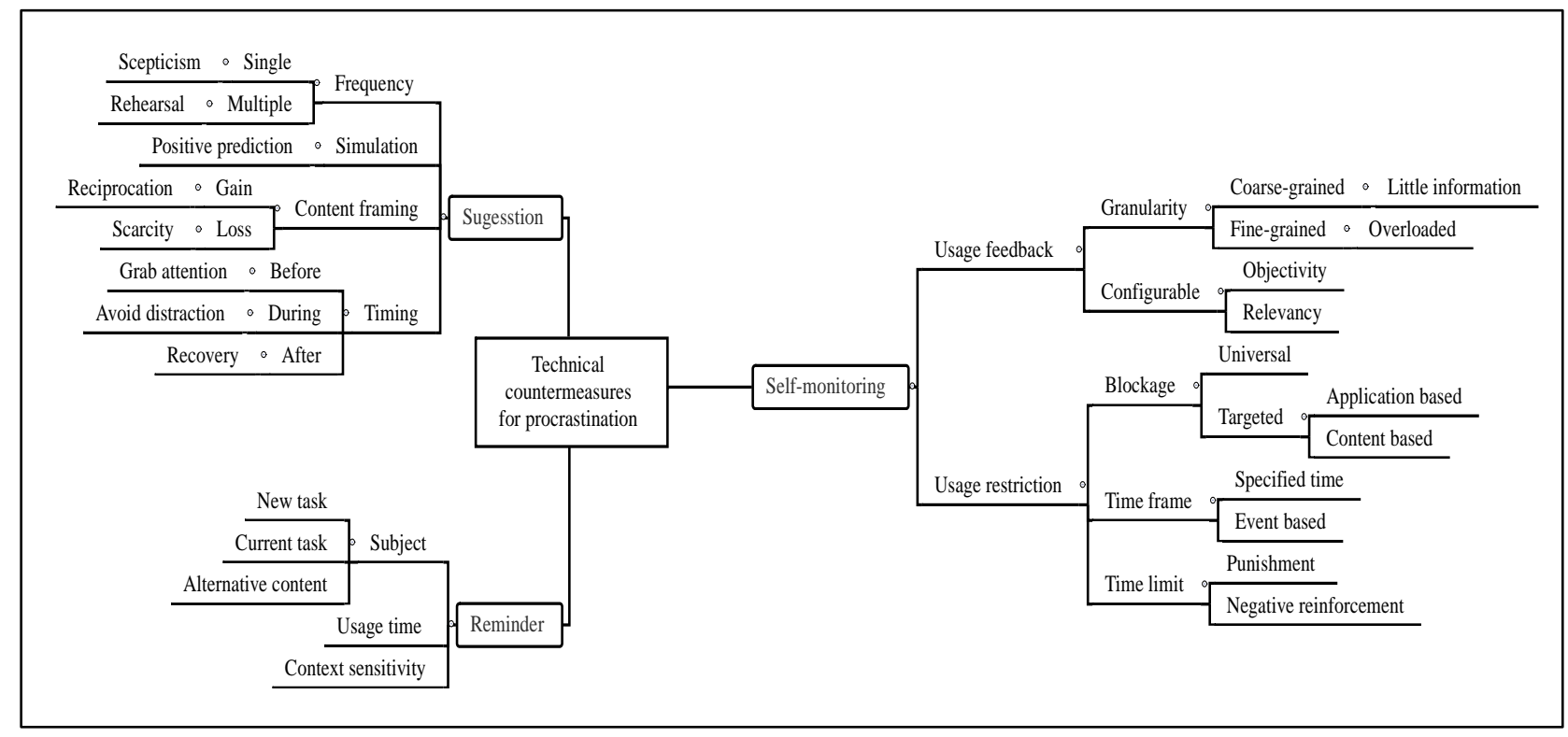

Fig.3: Procrastionation on SNS: technical countermeasures

whether messages are strong enough to motivate users to stop procrastinating.

The participants suggested that users should have the ability to decide the best way to remind themselves about their procrastination, and the frequency of the reminders. Some users can stop procrastinating when they receive the first reminder, whereas other needs multiple reminders combined with an option to lock certain social media. A participant proposed that: "the number of reminders should be increased and the time between these reminders should be reduced if the user does not stop procrastinating." Also, when a user interacts with others, the remainder could be for both users to help them stop procrastinating. One participant said: "If I got a reminder while chatting, this could increase my stress to stop procrastinating because other users do not know about my procrastination." Therefore, reminding both users at the same time could help to reduce the pressure that users feel when they withdraw from the interaction. Fig. 1 presented an example of a chatting interface where both parties are shown the timeframe of the chat and its expected end.

2) Self-monitoring: Is one of the persuasive techniques that can be used to motivate users to reduce their usage of SNS which can lead to raising awareness about procrastination [25]. Self-monitoring is divided into two subcategories: usage feedback and usage restriction.

a) Usage feedback: In this category, users can be provided with details about their usage to increase their awareness of procrastination. Based on these details, users can see how procrastination might harm their productivity, how often they become distracted while increasing user attention to the importance of regulating their SNS usage. Users can receive fine-grained or coarse-grained usage details. Finegrained usage data provides richer feedback concerning the type of applications being used, the length of usage, user location and interrupted activities while procrastinating. The coarse-grained format provides fewer details. These include general usage about SNS and which apps dominate user usage. However, the participants proposed to have configurable feedback, where users can choose the level of details in the feedback. This can help to get both objective and relevant data and avoid abundance in data leading to distraction and information overload. In contrast, the lack of feedback on user procrastination might prevent users from taking informed action to change their usage style. This suggests conflicting requirements in users around the desire to have brief information and at the same time be able to take a fully informed decision.

b) Usage Restrictions: Usage restrictions refer to the techniques that can be used to prevent or complicate users' access to SNS once they fail to meet the user restriction conditions. Usage restrictions are divided into three subcategories: blockage, timeframe, and time limit. Blockage techniques prevent users from accessing SNS once they procrastinate and deviate from their original tasks. The blockage can be universal for all SNS accounts or could be targeted at a specific application or the content that users view while procrastinating.

Another type of usage restriction is the timeframe which represents the period the user has to work on their original tasks. The timeframe can be specified time or event based. Hence, users are not allowed to access SNS during a given timeframe and will not be able to receive any notifications. Users can decide the time limit they wish to set for SNS access, and once exceeded their usage will be limited. This technique can help users to set goals and rehearse them to increase the control they have over their usage. The time limit technique can punish users and sign them out, thereby requiring signing in again after a while. However, negative reinforcement can also help to prevent users from accessing SNS for a limited time.

The modality of self-monitoring techniques can allow users to decide what kind of monitoring styles and tools they would 
like to use in order to reduce potential side-effects resulting from using the feedback and time restriction techniques. Some users can have more controllers over their usage so they just need to know certain information and feedback about their usage. However, some users may experience difficulty in stopping their procrastination when utilising their usage feedback due to their low self-control. Therefore, further actions, such as restricting them from browsing SNSs for some time, will be needed to help them stop procrastinating. One participant said: "preventing me from accessing SNSS during the class is more helpful because I cannot stop procrastinating by my own".

3) Suggestion technique: Suggestion refers to the informative process that shall help people in their thoughts, feelings, behaviour and decisions [28]. Suggestion can affect how people remember things and even how they act or behave based on the expected response. The expected response refers to when someone expects something to happen; their behaviour and reactions will contribute to matching that prediction [29]. For example, if an introvert student expects that rehearsing their presentation in front of the mirror will increase their confidence during the actual presentation, this expectation may play a significant role in increasing their confidence. However, the use of technology to deliver different kinds of suggestions can encourage users to follow these suggestions by being proactive and data-driven [25]. The suggestion can be more powerful when it causes users to do a series of actions through incremental suggestions rather than one suggestion [30]. When implementing the suggestion techniques, the design process should consider four factors: frequency of suggestions, simulation, content framing, and timing of the suggestion.

a) Suggestion frequency: The number of times users can receive suggestions might have a significant effect on encouraging them to follow the suggestion and reduce the likelihood of procrastinating. Some participants claim that multiple suggestions can increase their worry to stop procrastinating. For example, one participant said: "I usually ignore the suggestion the first time like I do with my alarm every morning by pressing snooze." In contrast, others proposed that a single suggestion works for most people because they take it seriously from the first time since they know there is no possibility of receiving more suggestions. Therefore, a single suggestion can increase user scepticism whereby users are afraid of not receiving more suggestions. In contrast, multiple suggestions can utilise rehearsal as a technique providing them with scenarios to practice and get them ready for performing the task at hand and also their usage control tactics, e.g. rehearsal on how to tell friends online in advance about time limitation and the framing and phrasing of the language used when opting out from an online dialogue and interaction.

b) Simulation: Refers to the scenarios of usage suggested to the user to see and reflect on how procrastination happens and its effects and alternative usage styles. This shall allow users to think on their own procrastination and make a selfassessment of it and its harm. The content of the messages can be textual in the form of certain sentences that have a strong inspirational effect, aimed at keeping users motivated; alternatively, it is possible to use images instead of text. For example, a metaphor like a tree can be shown to reflect adherence to plans with green leaves for being on time and brown and green leaves to reflect degrees of deviation. Hence, when users are presented with simulation, they can visualise in a more user-friendly way their status around procrastination and predict the outcome and perhaps change their strategies.

c) Suggestion framing: Refers to how suggestion content is phrased and the tone used to it. Some people prefer to know that harm and loss caused if they do not follow a recommended practice. Others refer a framing where they can be presented with the benefits that can be gained from following the suggestion. Hence, the suggestions can be lossframed or gain-framed. Gain framing focuses on the good things that can happen if users follow the suggestions. Cialdini demonstrated that the rule of reciprocation is to try to repay what other people and actions provided to us [31]. Hence, when a user knows that they will achieve something once they follow the suggestion, they are more likely to behave based on the suggestion. For example, if the users do not procrastinate when preparing for an exam, they will have a better opportunity to get a good mark. In contrast, loss framing focuses on the negative things that will happen if users do not follow the suggestions. For instance, users who do not attend lectures might fail the exam. Therefore, framing the messages that users receive in the suggestion can affect their behavioural decisions and encourage them to follow the suggestions. According to the prospect theory, people's choices are sensitive to how information is framed [32]. Hence, the scarcity principle suggests that people will follow the suggestion when they feel they have limited resources or options and they can be lost if they do not [31]. The framing of the message can also exploit this by showing people the opportunities and their time-limited nature.

d) The timing of suggestions: One of the important factors that were emphasised by the participants is whether users should receive suggestions before, during or after the original tasks that need to be performed. The suggestion can be sent before the task starts to grab the user's attention, and hence lead the user to focus fully on the tasks. Similarly, users can receive suggestions during the task when they receive an ad-hoc interaction or when they try to use SNS so that they remain motivated and avoid getting distracted that can result in procrastination. Furthermore, once a task has been completed, users can receive suggestions for a recovery time to refresh their mood and prepare them for any upcoming tasks and also to positively reinforce their achievement.

In terms of the modality of suggestion, different approaches were proposed concerning how participants should receive suggestions. About the frequency of the suggestions, users could receive single suggestions when they tend to procrastinate, with the number of suggestions increasing based on their level our duration of procrastination. For example, if they ignore the first suggestion, they then receive another suggestion with the same or different content. Receiving multiple suggestions can influence users to stop procrastinating and get ready for their original tasks. Of course, such suggestions should not in themselves become a nuisance and users can have the option to decide what type of 
content they would like to receive. This can influence their commitment to the decisions they made earlier when they designed the suggestion [31]. Framing the content of the suggestion can take different approaches. Some participants prefer to receive the suggestion in red to highlight the importance of the suggestion. For example, one participant said: "the suggestions should be in red to attract the user's attention." Also, some participants suggested that the suggestion could also be used to remind users to set their goals. Hence, based on the goals that users want to accomplish, the suggestion countermeasure can integrate with these goals and send motivational suggestions to help users continue their progress.

\section{Task motivations}

In this section, we discuss how users can be motivated to continue working on their tasks before the tendency to procrastinate takes place. Based on our study findings, task characteristics were found to be one of the factors that usually affect the tendency to procrastinate on SNSs. Task-related procrastination happens when users procrastinate because of a lack of motivation or the difficulty of the task whereby users avoid performing a task due to their fear of failure. Therefore, gamifying tasks can be a solution to enhance a user's motivation to complete them. Task motivations were divided into three sub-categories: commitment, Reward and milestones (see Fig.4).

1) Task commitment: Refers to the ability of an individual to demonstrate a focus on undertaking tasks for some time without distraction [33]. Renzulli claimed that it is not possible for an individual to experience high achievement without high task commitment. There is an associated relationship between task commitment and job performance which means that when users have a high commitment to their tasks, this can reduce the possibility of procrastination [34]. Furthermore, users might tend to lose their commitment when they have doubts about their ability to complete the task successfully or have a fear of evaluation which can negatively impact their task commitment [35, 36]. The commitment to a task can be driven by the user's motivation to perform the task. According to self-determination theory, users should have three basic psychological needs: autonomy, competence and relatedness [37]. Therefore, to motivate sufficient commitment should satisfy, to varying extents, those three needs. Furthermore, when users demonstrate their task commitment either to themselves or via a public commitment, this appears to have a positive impact on task completion [31].

2) Task reward: It is one of the factors that can motivate users to perform the task. For example, when the task has a sizeable reward, either intrinsic or extrinsic, this reward can have a significant effect in terms of increasing the user's enthusiasm to perform the task. The participants of the design sessions suggested that users could even be motivated by simple rewards such as adding points to some tally once users complete a task. Similarly, during the task, some form of motivational feedback was also considered to be useful. Such feedback can help users to maintain their progress and to ensure that they will continue to progress and the motivation will not be affected by the long time spent progressing the work. One participant said: "when the deadline is far away from the starting point, my motivation starts to decrease". Furthermore, the feedback that the user receives when working can also be personalised, to reflect their progress (see 3 ), to increase its effectiveness, and ensure that they remain motivated to complete their tasks.

3) Task milestone: Describes the tracking of the task's progress to accomplish them. Dividing tasks into different milestones can help to manage better the progress of the task which helps to ensure the quality of the work by reflecting it in different phases [38]. Furthermore, when users accomplish each phase, they can be rewarded to motivate them to complete the new phase. When the task is reduced into small phases and each phase can have a timeline to be monitored, this can help users to keep their progress under control and track their performance which decreased the chance of procrastination [39]. A task's milestones also help users to build their self-esteem especially when they get motivating feedback. The information about progress feedback can help users to reflect on their performance and planning and have clear ideas about the next stages before the completion of the task [40]. Control theory suggests that people can change their practice when there is a gap between the goal they would like to achieve and their current behaviour to minimise that gap [41]. The feedback that users receive can help to guide and motivate users to accomplish their goals and minimise the gap between their current behaviour and their desired behaviour [42].

The modality of task motivation is around how to gamify the commitment of the task which can help to keep users motivated to complete their original tasks and reduce the possibility of procrastination. In the design sessions, participants suggested different techniques to motivate users to complete their tasks successfully. These techniques include task reduction and reward. The reduction techniques refer to the action of dividing the task in smaller parts. The reduction can help to reduce the difficulty of the task that users might face when they deal with a particularly large task. Providing users with a progress bar was one of the most important strategies suggested by the participants. One participant said: "while I am performing a task I feel less motivated to complete it because I do not know how long I need to finish".

Task reward is another technique that users wanted to help combat procrastination. The reward can be either during the task or after the task has been completed. During the task, users can be rewarded for each milestone they achieve such as a reminder about the work that has been done. One participant said: "users can be thanked for the work they have achieved and motivated to complete the rest, such as 'well-done, you've done $70 \%$ of the work and still need to do $30 \%$ ". This kind of reminder triggers users' extrinsic motivation by appreciating their work so that they can feel motivated to accomplish the whole task. Another type of rewards that have been proposed by the participants is to compare users' progress with that of their colleagues by showing how many points' users have gained or the number of tasks that have been accomplished. Comparing users' achievement can increase competition between users which can increase their commitment to tasks and reduce the possibility of procrastinating. Also, some 


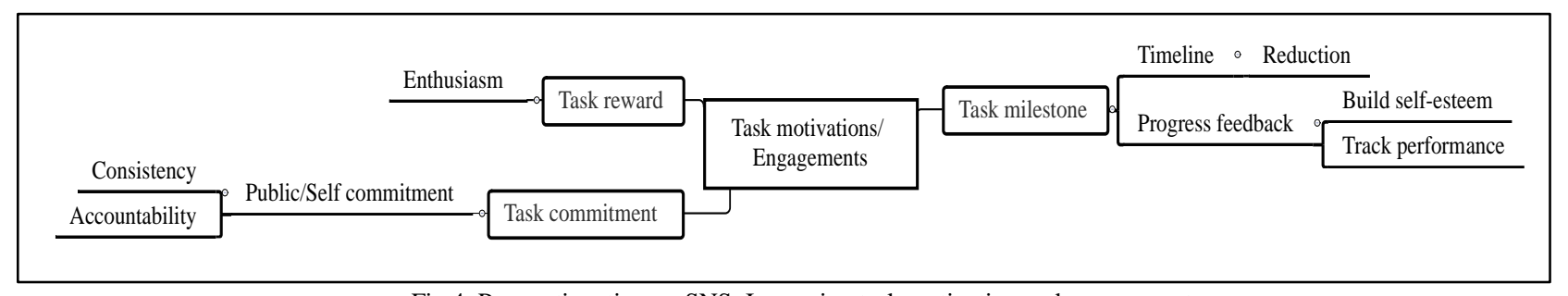

Fig.4. Procrastionation on SNS: Increasing task motivation and engagement

participants suggested that the task reward should be personalised for each user which could have a significant effect on an individual's motivation. Personalisation refers to the adaption of the output to the individual user instead of treating a group of people [25].

\section{USERS REQUIREMENTS VS EXISTING TOOLS}

Our suggested countermeasures are meant to help users to reduce and regulate procrastination. However, they may also come with their own risks and side-effects. This also applies to de-facto tools provided by the industry. For example, in iOS Downtime $^{1}$, users can schedule a time for muting the phone and avoiding notifications and their distraction. The downtime tools can introduce a risk whereby users keep thinking about whether they have received important notifications; i.e., hidden procrastination and fear of missing out. Hence, such tools should come with richer settings allowing people to decide upon alternative and socio-technical measures such as the one we proposed around suggestion, rehearsal and simulation. Another example from the iOS Downtime tool, users only have the option to select one block time in which they will be unable to receive any notifications. Table I presents a comparison between our proposed procrastination countermeasures in the area of usage regulation and the $\mathrm{iOS}$ Downtime tool.

Our suggested techniques and countermeasures allow users to customise the blockage time tool and what to block based on the types of the app and the content of interaction and to be integrated with their own calendar. This allows for greater personalisation and context sensitivity and increases acceptance and relevance. However, this also comes with additional costs in terms of requiring users to set up plans. There is a need for further research to ascertain the readiness of users to take the time to set up such plans, and how they perceive the cost/benefits of the additional effort. We expect this to also relate to several factors such as their technical skills and confidence and their perception of the need to make behavioural changes.

To empower existing tools, our findings suggested that online time management should be more fine-grained and sensitive to the content of the usage, its context and user status. The autoreply suggested in this paper has a richer format and setting to those tools in current communication software. Our auto-reply is intended to support the broader aim of managing user's expectations; both the sender and the receiver in an informative personalised style. We view procrastination as a social pressure problem in part. Importantly, our tools are suggested to reduce the pressure on all communicating parties.
We discussed the countermeasures and their modalities of application with the aim of reducing their potential negative side-effects. In our future work, we will use this knowledge to develop a method to help users identify the features and usage styles of SNS that trigger their procrastination and choose and configure suitable countermeasures that can help them to gain more control over their procrastination. We expect this method to be based on persuasive design and personalisation as we also acknowledge the role of personality traits, culture and self-control in the acceptance and efficiency of such tools.

TABLE I: DOWNTIME IN IOS SCREEN TIME VS OUR RECOMMENDATIONS

\begin{tabular}{|c|c|c|}
\hline $\begin{array}{c}\text { Comparison } \\
\text { criteria }\end{array}$ & $\begin{array}{c}\text { Current } \\
\text { Downtime iOS } \\
\text { Screen Time }\end{array}$ & Suggested design \\
\hline $\begin{array}{c}\text { Frequncy of } \\
\text { time } \\
\text { blockage }\end{array}$ & $\begin{array}{c}\text { Enable users to } \\
\text { only have one } \\
\text { time block }\end{array}$ & $\begin{array}{c}\text { Enable users to have more than one } \\
\text { time block, i.e. schedules and } \\
\text { timeframes }\end{array}$ \\
\hline $\begin{array}{c}\text { Modality of } \\
\text { setting time } \\
\text { blockage }\end{array}$ & $\begin{array}{c}\text { The user sets } \\
\text { the time } \\
\text { manually }\end{array}$ & $\begin{array}{c}\text { Manual and automated based on } \\
\text { users' calendar and user context }\end{array}$ \\
\hline $\begin{array}{c}\text { User status } \\
\text { unaware of the } \\
\text { receiver } \\
\text { availability }\end{array}$ & $\begin{array}{c}\text { Senders are aware of the receiver's } \\
\text { availability, meaning that the } \\
\text { receiver can have less pressure to } \\
\text { interact }\end{array}$ \\
\hline $\begin{array}{c}\text { Effectivness } \\
\text { on managing } \\
\text { others } \\
\text { expectations }\end{array}$ & $\begin{array}{c}\text { It does not } \\
\text { manage } \\
\text { senders' } \\
\text { expectations }\end{array}$ & $\begin{array}{c}\text { It contains an auto-reply to manage } \\
\text { senders' expectations }\end{array}$ \\
\hline $\begin{array}{c}\text { Stakeholders } \\
\text { Only focuses on } \\
\text { the relationship } \\
\text { between the } \\
\text { user and the } \\
\text { device }\end{array}$ & $\begin{array}{c}\text { Focus on the relation among the } \\
\text { user, the device and other contacts }\end{array}$ \\
\hline
\end{tabular}

Summary of what users expect from the antiprocrastination tools to be introduced to future social media:

- Making users more aware of how procrastination harms their productivity

- $\quad$ Guiding users to understand their current situation by providing them with feedback and guidance on how to gain more control over their procrastination.

- Having multiple stages to achieve results; helping users to control their procrastination in different stages such as feedback, a more advanced stage with reminders, and then the use of restrictions if required.

- Flexibility to enable users to decide the suitable techniques to use to avoid negative side-effects such as reactance. We recognise the risk of bias here. 
- Reducing the pressure that users feel to respond to notifications while working on their tasks by making other contacts aware of their availability.

- Managing other contacts' expectations, e.g. by showing them when they will receive a response and the time remaining for a chat.

- Supporting and motivating users to complete their original tasks; for instance, using task reduction and progress visualisation.

- Gamifying the commitment to the task to bring the fun that users experience on SNS into the work task environment.

\section{CONCLUSION}

In this paper, we have sought to gain a better understanding of user needs and how users could combat their procrastination. We also sought to explore the modality of the countermeasures which can be used to combat procrastination on SNS. The study highlighted the countermeasures that need to be considered as part of the future design of SNS to combat user procrastination. Some of these countermeasures are already incorporated into emerging tools such as iOS Screen Time and Google Digital Wellbeing to help users to manage their online time. However, these techniques focus mainly on the interaction between the user and the device; e.g. usage limits and feedback. This study has shown that some users procrastinate because of the pressure they feel when they become distracted, which in turn forces them to respond to SNS prompts, in order to develop or maintain a positive selfimage. In doing so, the study paid particular attention to the inclusion of three aspects of countermeasures: the user, the device, and society. This can help to reduce the pressure that leads users to procrastinate and helped them to gain greater control over their usage.

\section{REFERENCES}

[1] K. B. Klingsieck, "Procrastination: When good things don't come to those who wait," European Psychologist, vol. 18, p. 24, 2013.

[2] L. Ivarsson and P. Larsson, "Personal Internet usage at work: A source of recovery," Journal of Workplace Rights, vol. 16, 2011.

[3] K. R. Kim and E. H. Seo, "The relationship between procrastination and academic performance: A meta-analysis," Personality and Individual Differences, vol. 82, pp. 26-33, 2015.

[4] D. Blackwell, C. Leaman, R. Tramposch, C. Osborne, and M. Liss, "Extraversion, neuroticism, attachment style and fear of missing out as predictors of social media use and addiction," Personality and Individual Differences, vol. 116, pp. 69-72, 2017.

[5] B. J. Fogg, "Persuasive technology: using computers to change what we think and do," Ubiquity, vol. 2002, p. 5, 2002.

[6] S. Deterding, D. Dixon, R. Khaled, and L. Nacke, "From game design elements to gamefulness: defining gamification," in Proceedings of the 15th international academic MindTrek conference: Envisioning future media environments, 2011, pp. 9-15.

[7] G. Drosatos, F. Nalbadis, E. Arden-Close, V. Baines, E. Bolat, L. Vuillier, T. Kostoulas, S. Wasowska, M. Bonello, J. Palles, J. McAlaney, K. Phalp, and R. Ali, "Empowering Responsible Online Gambling by Real-time Persuasive Information Systems," 2018 12th International Conference on Research Challenges in Information Science (RCIS), 2018.

[8] Z. Fitz-Walter, D. Tjondronegoro, and P. Wyeth, "Orientation passport: using gamification to engage university students," in Proceedings of the 23rd Australian computer-human interaction conference, 2011, pp. 122125.

[9] J. Hamari, J. Koivisto, and H. Sarsa, "Does gamification work?--a literature review of empirical studies on gamification," in 2014 47th Hawaii international conference on system sciences (HICSS), 2014, pp. 3025-3034.

[10] A. Shahri, M. Hosseini, M. Almaliki, K. Phalp, J. Taylor, and R. Ali, "Engineering software-based motivation: a persona-based approach," in Research Challenges in Information Science (RCIS), 2016 IEEE Tenth International Conference on, 2016, pp. 1-12.

[11] A. Algashami, S. Cham, L. Vuillier, A. Stefanidis, K. Phalp, and R. Ali, "Conceptualising Gamification Risks to Teamwork within Enterprise," in IFIP Working Conference on The Practice of Enterprise Modeling (PoEM), 2018, pp. 105-120.

[12] A. Shahri, M. Hosseini, K. Phalp, J. Taylor, and R. Ali, "Towards a code of ethics for gamification at enterprise," in IFIP Working Conference on The Practice of Enterprise Modeling (PoEM), 2014, pp. 235-245.

[13] E. B.-N. Sanders and P. J. Stappers, "Co-creation and the new landscapes of design," Co-design, vol. 4, pp. 5-18, 2008.

[14] J. Castillo, "Research Population: Retrieved 17 Jun, 2012 from Experiment Resources: http:/www," Experiment resources. com/researchpopulation. html, 2009.

[15] R. C. Fraley and N. W. Hudson, "Review of intensive longitudinal methods: an introduction to diary and experience sampling research," ed: Taylor \& Francis, 2014.

[16] A. F. Payne, K. Storbacka, and P. Frow, "Managing the co-creation of value," Journal of the academy of marketing science, vol. 36, pp. 83-96, 2008.

[17] J. H. Song and C. R. Adams, "Differentiation through customer involvement in production or delivery," Journal of Consumer Marketing, vol. 10, pp. 4-12, 1993.

[18] V. Braun and V. Clarke, "Using thematic analysis in psychology," Qualitative research in psychology, vol. 3, pp. 77-101, 2006.

[19] E. A. Locke and G. P. Latham, A theory of goal setting \& task performance: Prentice-Hall, Inc, 1990.

[20] S. Consolvo, P. Klasnja, D. W. McDonald, and J. A. Landay, "Goalsetting considerations for persuasive technologies that encourage physical activity," in Proceedings of the 4th International Conference on Persuasive Technology, 2009, p. 8.

[21] R. S. Rubin, "Will the real SMART goals please stand up," The Industrial-Organizational Psychologist, vol. 39, pp. 26-27, 2002.

[22] E. Z. Rothkopf and M. Billington, "Goal-guided learning from text: inferring a descriptive processing model from inspection times and eye movements," Journal of Educational Psychology, vol. 71, p. 310, 1979.

[23] T. H. Macan, C. Shahani, R. L. Dipboye, and A. P. Phillips, "College students' time management: Correlations with academic performance and stress," Journal of Educational Psychology, vol. 82, p. 760, 1990.

[24] T. Walter and A. Siebert, Student success: How to do better in college and still have time for your friends: Holt, Rinehart and Winston, 1981.

[25] H. Oinas-Kukkonen and M. Harjumaa, "Persuasive systems design: Key issues, process model, and system features," Communications of the Association for Information Systems, vol. 24, p. 28, 2009.

[26] I. Ajzen, "The theory of planned behavior," Organizational behavior and human decision processes, vol. 50, pp. 179-211, 1991.

[27] A. Bandura, "Self-efficacy: toward a unifying theory of behavioral change," Psychological review, vol. 84, p. 191, 1977.

[28] S. B. Hall, "Theories Of Suggestion," British Medical Journal, vol. 1, p. $158,1928$.

[29] D. Albarracin and R. S. Wyer Jr, "The cognitive impact of past behavior: influences on beliefs, attitudes, and future behavioral decisions," Journal of personality and social psychology, vol. 79, p. 5, 2000.

[30] A. P. Mathew, "Using the environment as an interactive interface to motivate positive behavior change in a subway station," in $\mathrm{CHI} 05$ Extended Abstracts on Human Factors in Computing Systems, 2005, pp. $1637-1640$

[31] R. B. Cialdini, Influence vol. 3: A. Michel Port Harcourt, 1987. 
[32] A. Tversky and D. Kahneman, "The framing of decisions and the psychology of choice," science, vol. 211, pp. 453-458, 1981.

[33] J. S. Renzulli, E. J. Gubbins, K. S. McMillen, R. D. Eckert, and C. A. Little, Systems and models for developing programs for the gifted and talented: Creative Learning Press Mansfield Center, CT, 1986.

[34] C. Vandenberghe, K. Mignonac, and C. Manville, "When normative commitment leads to lower well-being and reduced performance," Human relations, vol. 68, pp. 843-870, 2015.

[35] C. Senecal, K. Lavoie, and R. Koestner, "Trait and situational factors in procrastination: An interactional model," Journal of social Behavior and personality, vol. 12, p. 889, 1997.

[36] E. H. Seo, "The relationship of procrastination with a mastery goal versus an avoidance goal," Social Behavior and Personality: an international journal, vol. 37, pp. 911-919, 2009.

[37] R. M. Ryan and E. L. Deci, "Self-determination theory and the facilitation of intrinsic motivation, social development, and well-being," American psychologist, vol. 55, p. 68, 2000.
[38] S. Srivannaboon, "Achieving competitive advantage through the use of project management under the plan-do-check-act concept," Journal of General Management, vol. 34, pp. 1-20, 2009.

[39] S. Kambhampati, "A comparative analysis of partial order planning and task reduction planning," ACM SIGART Bulletin, vol. 6, pp. 16-25, 1995.

[40] D. J. Nicol and D. Macfarlane-Dick, "Formative assessment and selfregulated learning: A model and seven principles of good feedback practice," Studies in Higher Education, vol. 31, pp. 199-218, 2006.

[41] C. S. Carver and M. F. Scheier, "Control theory: A useful conceptual framework for personality-social, clinical, and health psychology," Psychological bulletin, vol. 92, p. 111, 1982.

[42] E. L. Larson, S. J. Patel, D. Evans, and L. Saiman, "Feedback as a strategy to change behaviour: the devil is in the details," Journal of evaluation in clinical practice, vol. 19, pp. 230-234, 2013. 